\title{
Some low-temperature properties of a generalized Hubbard model with correlated hopping
}

\author{
L. Didukh, V. Hankevych ${ }^{1}$, Yu. Skorenkyy \\ Ternopil State Technical University, Department of Physics, 56 Rus'ka Str., Ternopil UA-46001, Ukraine
}

\begin{abstract}
In the present paper we study some correlation effects in a generalized Hubbard model with correlated hopping within low-temperature region using a generalized mean-field approximation. It is shown that in a series of cases the model leads to consequences deviating essentially from those of the Hubbard model. We consider the possibility of applying the result to interpret the peculiarities of physical properties of systems with narrow energy bands.
\end{abstract}

Keywords: Correlated hopping; the Hubbard model; quasiparticle energy spectrum.

Theoretical analyses, on the one hand, and available experimental data, on the other hand, point out the necessity of the Hubbard model generalization by taking into account correlated hopping $[1,2]$. In the recent few years, similar models have been studied intensively (e.g., see Refs. [3] and references therein). In particular, some of these models [4] have been solved exactly in a wide range of parameters.

The present paper is devoted to study of correlation effects in a generalized Hubbard model with correlated hopping within low-temperature region in the case of partially filled narrow energy bands.

We start from the generalization of the Hubbard model with correlated hoppings $T_{1}$ and $T_{2}$ [1]:

$$
H=-\mu \sum_{i \sigma} a_{i \sigma}^{+} a_{i \sigma}+t(n) \sum_{i j \sigma}^{\prime} a_{i \sigma}^{+} a_{j \sigma}+
$$

\footnotetext{
1 Corresponding author. E-mail: vaha@tu.edu.te.ua
}

$$
T_{2} \sum_{i j \sigma}^{\prime}\left(a_{i \sigma}^{+} a_{j \sigma} n_{i \bar{\sigma}}+\text { h.c. }\right)+U \sum_{i} n_{i \uparrow} n_{i \downarrow},
$$

where all the notations are usual, $t(n)=t_{0}+n T_{1}$, $t_{0}$ is the nearest-neighbor hopping integral, $n$ is the electron concentration, the prime at the sums signifies that $i \neq j$.

The peculiarity of model (1) is taking into account the correlated hopping $T_{1}$ leading to the concentration dependence of the hopping integral $t(n)$ in contrast to similar models which are considered in Refs. [3].

In Ref. [5] we have obtained the single-particle Green function and the quasiparticle energy spectrum (which has the exact atomic and band limits) for arbitrary electron concentration using a variant [6] of the generalized mean-field approximation [7]. For the case $n=1$ and $t(n)=-T_{2}$ the approach recovers (see Ref. [8]) the exact results [4].

The energy gap $\Delta E$ (which is calculated from the spectrum) is found to increase with a deviation 
from half-filling ( $\Delta E$ has a minimum at $n=1$ ). This explains the fact that in a metallic phase the vanadium oxides with fractional number of electrons per cation $\left(\mathrm{V}_{k} \mathrm{O}_{2 k-1}, k \geq 3\right)$ exhibit "metallicity" to lesser extent than the compounds $\mathrm{VO}_{2}$ and $\mathrm{V}_{2} \mathrm{O}_{3}$ (integer number of electrons per site) [9].

Within low-temperature region for $U \rightarrow \infty$ width of the lower Hubbard band at $n<1$ is $W_{l}=$ $2 w[2 /(2-n)-n]$; and at $n>1$ width of the upper Hubbard band is $W_{u}=2 \tilde{w}(n-2+2 / n)$, where $w=z|t(n)|, \quad \tilde{w}=z|\tilde{t}(n)|, \tilde{t}(n)=t(n)+2 T_{2}, \quad z$ is the number of nearest neighbors to a site.

The concentration dependence of subband widths plotted in Fig. 1 is caused firstly, by correlation effect of narrowing of subbands, secondly, by the concentration dependence of the hopping integrals in the lower $t(n)$ and upper $\tilde{t}(n)$ Hubbard bands (this is a peculiarity of the present model). Taking into account correlated hopping leads to the essential narrowing of subband, and what is more, narrowing by this factor increases with increasing of electron concentration. Fig. 1 illustrates the non-equivalence of the cases $n<1$ and $n>1$ in the framework of the present model (in contrast to the electron-hole symmetry of the Hubbard model). One can see that at some values of the parameters $\tau_{1}=T_{1} /\left|t_{0}\right|, \tau_{2}=T_{2} /\left|t_{0}\right|$ correlated hopping $W_{l} \gg W_{u}$, thus effective mass of current carriers within the lower Hubbard band $m_{l} \ll m_{u}$ ( $m_{u}$ is the effective mass of current carriers within the upper Hubbard band). So, the narrow-band materials with conductivity determinated by current carriers within the lower band can have much smaller resistivity than the compounds with conductivity determinated by current carriers within the upper band. This fact is confirmed by the experimental data [10]: the metalooxides with less than half-filled $3 d$-shell $\left(\mathrm{Mn}_{2} \mathrm{O}\right)$ exhibit much higher conductivity than the compounds with half or more than half-filled $3 d$-shell (MnO, $\mathrm{NiO})$.

V.H. thanks the Organizing Committee of the LT22 Conference for a grant. The authors are grateful to Prof. D. Khomskii for valuable discussions on the part of the result considered in the

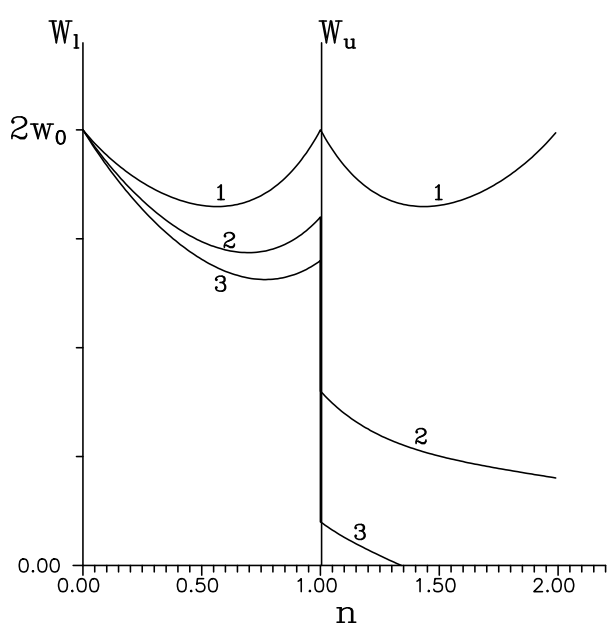

Fig. 1. Width of the lower $\left(W_{l}\right)$ and upper $\left(W_{u}\right)$ bands as a function of electron concentration $n: 1) \tau_{1}=\tau_{2}=0$ (the Hubbard model); 2) $\tau_{1}=\tau_{2}=0.2$; 3) $\tau_{1}=\tau_{2}=0.3$.

present work.

\section{References}

[1] L.D. Didukh, Fiz. Tverd. Tela 19, 1217 (1977) [Sov. Phys. Solid State 19, 711 (1977)].

[2] J.E. Hirsh, Physica C 158, 326 (1989); Physica B 199200, 366 (1994).

[3] J.C. Amadon and J.E. Hirsch, Phys. Rev. B 54, 6364 (1996); A.A. Aligia et al., Eur. Phys. Journ. B 5, 371 (1998); B.R. Bułka, Phys. Rev. B 57, 10303 (1998).

[4] R. Strack, D. Vollhardt, Phys. Rev. Lett. 70, 2637 (1993); A.A. Ovchinnikov, J. Phys.: Condens. Matter 6, 11057 (1994); A.A. Aligia et al., Phys. Rev. B 51, 13774 (1995).

[5] L. Didukh, V. Hankevych, Fiz. Nizkikh Temp. 25, 481 (1999) [Low Temp. Phys. 25, 354 (1999)].

[6] L. Didukh, phys. stat. sol. (b) 206, R5 (1998).

[7] L.M. Roth, Phys. Rev. 184, 451 (1969); D.N. Zubarev, Yu.G. Rudoj, Uspekhi Fiz. Nauk 163, 103 (1993) [Phys. Usp. 36, 744 (1993)].

[8] L. Didukh and V. Hankevych, phys. stat. sol. (b) 211 , 703 (1999); L. Didukh, V. Hankevych, Yu. Dovhopyaty, Physica B 259-261, 719 (1999).

[9] K. Kosuge et al., IEEE Trans. Mag. Mag-8, 581 (1972); R.O. Zaitcev et al., Uspekhi Fiz. Nauk 148, 603 (1986) [Sov. Phys. Usp. 29, 322 (1986)].

[10] G.H. Jonker, J. Phys. Chem. Solids 9, 165 (1959). 